\title{
Reflets
}

Revue ontaroise d'intervention sociale et communautaire

\section{Résumés des communications}

Caroline Andrew, Lyne Bouchard, Denyse Culligan, Denise Lemire, Natalie Beausoleil, Hélène Gendron, Denise Merkle, Gaëtane Pharand, Danielle Fournier, Lucie Massicotte, France Gélinas, Mary Carter, Hélène Dallaire, Lucie Plante, Colette Prévost, Lorraine LeBlanc, Manon Lemonde, Éva Mazerolle, Céline Simard, Anne Hodge, Diane Dupont, Marie-Béatrice Ngaba, Anne-Marie Mboma, Denyse Côté, Lise Ladouceur et Michelle Bernier-Wilson

Volume 3, numéro 2, automne 1997

Visibles et Partenaires : Pratiques et recherches féministes

URI : https://id.erudit.org/iderudit/026192ar

DOI : https://doi.org/10.7202/026192ar

Aller au sommaire du numéro

Éditeur(s)

Reflets : Revue ontaroise d'intervention sociale et communautaire

ISSN

1203-4576 (imprimé)

1712-8498 (numérique)

Découvrir la revue

Citer ce document

Andrew, C., Bouchard, L., Culligan, D., Lemire, D., Beausoleil, N., Gendron, H., Merkle, D., Pharand, G., Fournier, D., Massicotte, L., Gélinas, F., Carter, M., Dallaire, H., Plante, L., Prévost, C., LeBlanc, L., Lemonde, M., Mazerolle, É., Simard, C., Hodge, A., Dupont, D., Ngaba, M.-B., Mboma, A.-M., Côté, D., Ladouceur, L. \& Bernier-Wilson, M. (1997). Résumés des communications. Reflets, 3(2), 312-328. https://doi.org/10.7202/026192ar

Tous droits réservés (C) Reflets : Revue ontaroise d'intervention sociale et communautaire, 1997
Ce document est protégé par la loi sur le droit d'auteur. L'utilisation des services d'Érudit (y compris la reproduction) est assujettie à sa politique d'utilisation que vous pouvez consulter en ligne.

https://apropos.erudit.org/fr/usagers/politique-dutilisation/ 


\section{Résumés des communications}

Afin que les lectrices et les lecteurs de Reflets puissent connaître l'ensemble des thèmes qui ont été abordés lors du colloque Visibles et Partenaires et ainsi avoir un éventail plus complet des domaines de pratiques et de recherches concernant les femmes de l'Ontario français, nous avons pensé inclure les résumés des conférences qui n'ont pas fait l'objet d'un article pour ce numéro.

\section{Santé et services sociaux en Ontario français : pratiques des femmes}

par

Caroline Andrew, Lyne Bouchard, Denyse Culligan et Denise Lemive

Des membres d'une équipe de chercheures, constituée en 1995, en collaboration avec la Table féministe francophone de concertation provinciale de l'Ontario, présenteront les grandes lignes d'une recherche sur la santé et les services sociaux en Ontario français. Nous souhaitons informer le milieu féministe de notre recherche, sa problématique, sa démarche, ses attentes et, si possible, ses résultats préliminaires. Nous voulons profiter de cette occasion pour amorcer un dialogue entre les participantes à la conférence quant à la nature et la visibilité des pratiques des femmes francophones dans le domaine de la santé et des services sociaux. 


\section{Pratiques d'apparence, le rapport au corps et l'identité chez les femmes francophones en Ontario: premiers résultats de recherche}

par

\section{Natalie Beausoleil}

Cet exposé vise à faire part des premiers résultats d'une recherche sur l'importance de la culture et des questions identitaires chez les femmes francophones en Ontario. Cette recherche pose, entre autres, les questions suivantes. Comment la culture façonne-telle les corps des femmes francophones en Ontario? Est-ce que les femmes francophones en Ontario présentent leur corps comme signes distinctifs de la majorité anglo-saxonne, et si oui, comment le font-elles? Les données de cette recherche proviennent d'entrevues avec des femmes francophones des régions de Toronto, d'Ottawa et de Sudbury. J'examine de façon empirique comment une trentaine de femmes en Ontario, incluant des immigrantes francophones, produisent et perçoivent leur corps comme système de signes et élément central de l'identité individuelle et collective.

\section{Représentation médiatique des femmes}

\section{par}

\section{Hélène Gendron}

La première partie de la conférence portera sur le thème «visibles mais non partenaires». Cette partie présentera quelques modèles de femmes qui se sont distinguées au cours des années dans les professions relevant des médias et des communications. La seconde partie, "partenaires parce que visibles", permettra de voir la progression des femmes et les pressions qu'elles ont exercées pour 
en arriver à être reconnues. Les quelques succès qui seront illustrés dans cette communication ne signifient pas que la bataille des femmes dans les médias et les communications soit gagnée. L'évolution actuelle de la société vers la droite et vers des positions plus conservatrices exigent que nous demeurions vigilantes, que nous soyons à l'écoute et prêtes à dénoncer l'injustice où qu'elle se trouve, pour que nos filles et nos petites-filles n'aient pas à refaire le chemin parcouru, mais puissent continuer à progresser vers une égalité réelle dans la différence. Pour terminer, l'auteure discutera aussi de la visibilité des femmes dans les médias, des partenariats à développer et enfin de l'utilisation que les femmes francophones peuvent faire des médias afin de véhiculer leurs messages.

\section{Étude des besoins des femmes franco-africaines de Sudbury}

par

Marguerite Mbonimpa

Ce n'est pas par hasard que j'ai choisi ce sujet. Ce thème cadre bien avec mes préoccupations privées, mais il indique aussi une situation réelle, objective, vécue par un groupe précis de personnes. Depuis mon arrivée à Sudbury,j'ai constaté que les femmes francoafricaines qui résidaient dans cette ville, n'étaient impliquées, ni dans les activités socio-économiques, ni dans celles de la communauté des Franco-Ontariennes. Certaines questions me sont venues à l'esprit pour essayer de comprendre cet isolement. J'ai donc élaboré un questionnaire d'entrevue que j'ai soumis à cette clientèle-cible. L'analyse des réponses fournies m'a permis de relever un certain nombre des problèmes que rencontre ce groupe de femmes, et enfin de proposer un éventail de solutions. Le but de ma recherche était de voir comment on pourrait favoriser l'intégration des Franco-Africaines dans la communauté des femmes franco-ontariennes de Sudbury. On ne peut y parvenir 
que par la création des liens d'échanges, par l'information, la sensibilisation et la connaissance mutuelle à partir des codes culturels qui gouvernent les réactions et les comportements des unes et des autres.

\section{L'Union culturelle des Franco-Ontariennes affiche fièrement ses couleurs}

par

\section{Madeleine Paquette}

L'Union culturelle des Franco-Ontariennes (UCFO) est un organisme sans but lucratif qui se consacre à améliorer les conditions et les réalités sociales des femmes francophones de l'Ontario.Actuellement, l'UCFO a mis en marche deux importants projets. La Coop a pour mission d'ajouter une valeur à la production des artistes et artisanes de l'UCFO et de contribuer à accroître leur autonomie financière tout en leur donnant un véhicule de mise en marché de leur production. Quant à la Galerie, il s'agit d'un projet fondé dans le but de créer des pièces à l'effigie de sites franco-ontariens. Chaque pièce est faite et peinte à la main. Les profits de ce projet seront versés à la production d'autres pièces et vers le financement des activités de l'organisme. L'UCFO bénéficiera des retombées de ces projets dont une autonomie à long terme, une visibilité en tant qu'organisme et une augmentation du nombre de membres. Les membres démontrent un enthousiasme remarquable à l'égard de ces projets; elles en sont les pionnières. En général, il est juste de dire que les accomplissements de l'UCFO peuvent devenir une source d'inspiration pour la société. Tous les projets sont réalisés pour et par des femmes. Les membres de l'UCFOn'ont pas peur de prendre de l'initiative. C'est la seule façon de réussir de tels accomplissements. 


\section{Pleins feux sur la recherche-action : un bilan des partenaires}

par

Darquise Deschamps et Marie-Luce Garceau

La Fédération des femmes canadiennes-françaises de l'Ontario présentera un bilan des acquis des partenaires de la rechercheaction sur la situation des femmes francophones âgées de 45 à 64 ans qui vivent en Ontario. C'est à partir du point de vue des femmes qui ont participé à ce projet, pendant deux ans, que nous verrons les facteurs qui les ont motivées à investir temps et énergie dans cette étude. Nous toucherons aux avantages et aux désavantages à travailler avec des chercheures universitaires. Nous examinerons les problèmes concernant l'organisation et la communication entre les partenaires. Nous poserons un regard particulier sur les habiletés et les connaissances que les partenaires ont développées pendant la durée de ce projet. Finalement, nous nous attarderons sur les retombées de la recherche-action pour l'organisation et les partenaires.

\section{Retour au capitalisme impitoyable}

\section{par}

\section{Denise Merkle}

Il se peut que le «bon vieux temps», où les bonnes petites femmes au foyer cuisinaient le petit déjeuner à sept heures du matin, soit l'idéal de certains détenteurs du pouvoir à Toronto, comme celui de certains hommes politiques du camp républicain aux ÉtatsUnis. Mais nos voisines américaines craignant les effets néfastes de la position républicaine ont eu la perspicacité de la rejeter. Ici, par contre, la majorité des femmes et des hommes anglophones 
et francophones semblent avoir appuyé les Conservateurs de Mike Harris. Les décisions de ce gouvernement s'accordent avec la ligne de visée républicaine : législation en vue de l'équité en matière d'emploi supprimée, l'enseignement postsecondaire moins accessible et plus cher pour les femmes que pour les hommes grâce à la hausse des frais de scolarité, subventions destinées aux garderies sabrées, subventions destinées aux femmes et aux enfants agressés coupées, et j'en passe. Le parti conservateur est en train de défaire plus de trente ans de progrès social et les femmes francophones en souffrent beaucoup.

\section{Chantage et climat de travail empoisonné: le harcèlement au travail}

par

\section{Gä̈tane Pharand}

Dans cet atelier pratique, nous aborderons la définition du harcèlement au travail et des formes de harcèlement. Nous verrons comment il est possible de déterminer si une situation vécue en milieu de travail est du harcèlement et quels sont les recours des victimes pour lutter contre ce phénomène. Nous toucherons aux façons dont les femmes peuvent se protéger contre le harcèlement au travail, aux lois qui existent pour assurer cette protection et aux responsabilités des employeurs, travailleuses et travailleurs face au harcèlement. Finalement, les participantes auront également l'occasion de participer à des mises en situation de harcèlement pour tenter d'en cerner les enjeux. 


\section{Cuisines collectives : solidarité, entraide et lutte à la pauvreté}

par

\section{Danielle Fournier et Lucie Massicotte}

Les cuisines collectives existent au Québec depuis une dizaine d'années. Nées dans le quartier Hochelaga-Maisonneuve, elles se sont développées rapidement sur tout le territoire. Les cuisines regroupent principalement des femmes, le plus souvent mères de familles, exclues du marché du travail, qui se retrouvent une fois par mois pour cuisiner en commun afin d'économiser. La recherche-action, que nous achevons en partenariat avec le Regroupement des cuisines collectives du Québec et Relaisfemmes, a pour objectifs de dresser un portrait des cuisines collectives québécoises, d'en comprendre le fonctionnement et de décrire les caractéristiques des participantes. De plus, cette recherche permet de mieux cerner l'histoire des cuisines collectives et d'approfondir les liens existant avec les cuisines péruviennes. Dans cette communication, nous voulons rendre compte des principales conclusions de notre recherche et des acquis du voyage au Pérou. Les cuisines collectives se sont développées comme un instrument d'entraide et d'alternative au dépannage alimentaire. Bien qu'utilisées de diverses manières en intervention, la majorité des cuisines poursuivent ces objectifs. Les cuisines, souvent rattachées à des organismes communautaires, sont très souvent des lieux peu formalisés et peu institutionnalisés, qui restent très proches de l'univers domestique. Elles ont un fort potentiel de développement personnel et social pour les participantes; en fait, ce sont des lieux d'entraide et de solidarité, mais peu d'entre elles développent des initiatives reliées au développement économique communautaire. 


\section{Développement de partenariats}

par

\section{France Gélinas}

Dans cette conférence, j'aimerais passer en revue le mandat du Centre de santé communautaire de Sudbury en ce qui a trait aux femmes francophones. Le Centre de santé communautaire de Sudbury a comme mandat de desservir la population francophone du grand Sudbury. Il a donc identifié trois groupes cibles pour ses services. Les femmes sont un des trois groupes avec les aînées et aînés et les jeunes. Dans un deuxième temps, je discuterai des raisons qui soustendent l'établissement de partenariats. Quasi imposés par l'actuel gouvernement provincial, les partenariats se sont vite distingués par leurs habiletés à multiplier les résultats. En effet, le partenariat s'additionne ainsi : $1+1+1=10$. Mais, comment choisir ses partenaires? Qui se ressemble s'assemble n'est pas toujours la meilleure façon de faire. Quelles sont alors les stratégies et les étapes de développement des partenariats? Certains sont spontanés, d'autres se développent plus lentement. De plus, on trouve différents types de partenariats à tous les niveaux: administratif, fonctionnel, clinique ou dans les programmes. Or, il semble que les intervenants cliniques, habitués au travail en équipe multidisciplinaire, font une transition très naturelle vers le partenariat. Dans d'autres domaines, c'est beaucoup plus nouveau et moins bien compris. Finalement, quels sont les résultats? À quoi pouvons-nous s'attendre et comment pouvons-nous mesurer les résultats? Cette conférence sera truffée d'exemples vécus au Centre de santé communautaire de Sudbury. 


\section{Agression sexuelle : dissiper les mythes}

par

\section{Mary Carter}

Cette présentation débutera par une introduction sur l'agression sexuelle. J'offrirai d'abord une définition, puis je présenterai quelques statistiques et les lois qui régissent l'agression sexuelle. Par la suite, nous discuterons des faits afin de dissiper les mythes de l'agression. J'expliquerai le processus du programme offert à l'hôpital Général de Sudbury aux victimes d'agression sexuelle. Nous terminerons par une discussion sur la problématique.

\section{Défi de l'équité}

par

\section{Hélène Dallaire}

L'équité dans les cours d'éducation physique au niveau secondaire n'est pas la responsabilité unique des enseignants et enseignantes. Ainsi, une recherche-action réalisée par le Réseau national d'action éducation femmes auprès des jeunes francophones vivant en milieu minoritaire a permis d'obtenir les premières données canadiennes sur la problématique de l'équité en éducation physique. Les résultats obtenus par l'intermédiaire d'une méthodologie quantitative et qualitative seront présentés. De plus, la phase action de cette recherche aura permis d'identifier de multiples stratégies favorisant l'équité et s'adressant à une diversité de partenaires. L'équité n'est certainement pas la responsabilité unique des enseignantes et enseignants, pas plus qu'elle n'est l'équivalent de la mixité dans les cours d'éducation physique. 


\section{Harmonisation travail — famille}

par

\section{Lucie Plante}

L'augmentation sans cesse croissante de la présence des femmes sur le marché du travail amène un questionnement sur la situation du double emploi des femmes. La présente recherche étudie les moyens que les femmes francophones du Nord-Est de l'Ontario prennent pour combler leurs besoins. Comment se fait le partage des responsabilités familiales? Quelles stratégies sociales seraient nécessaires pour améliorer la situation des travailleuses? Autant de questions importantes qui ont amené le développement d'une recherche sur l'harmonisation entre le travail et la famille. La présentation mettra en lumière la démarche poursuivie, les forces et les limites de la recherche ainsi que les pistes de recherche à poursuivre.

\section{Du conservatisme au féminisme : place aux programmes féministes}

par

\section{Colette Prévost}

Traditionnellement, les organismes de services n'ont pas de philosophie féministe. En fait, ils semblent davantage se rapprocher d'une philosophie conservatrice qui, en somme, permet le maintien des services traditionnels. Cet atelier vise à partager l'expérience d'un organisme qui est passé d'une philosophie conservatrice à une philosophie féministe. Partant d'abord d'une structure hiérarchique embrassant les concepts conservateurs, nous verrons les différents problèmes qui se sont érigés dans la mise en place du féminisme. 


\section{Impact de la refonte des services et des soins de santé pour les personnes âgées}

par

Lorraine LeBlanc, Manon Lemonde et Éva Mazerolle

La discussion portera sur les conséquences engendrées par les remaniements au sein des services de santé destinés aux personnes âgées. En effet, l'abolition de la Loi sur la création d'organismes de services polyvalents, la modification de la Loi sur le remboursement des médicaments, la restructuration et le virage ambulatoire affectent spécifiquement les personnes âgées. Dans le Nord, les conséquences sont d'autant plus grandes que l'accès aux services de santé est déjà réduit. Il faut être sensible à l'impact des compressions budgétaires sur les personnes âgées, car elles forment un groupe économique considéré comme étant très vulnérable. En effet, elles ont beaucoup plus de difficultés à s'adapter aux changements d'attitude véhiculée par la restructuration. Cette nouvelle orientation va à l'encontre du système dans lequel ces personnes ont grandi, c'est-à-dire à l'intérieur d'une société qui assumait la responsabilité de leur santé grâce à l'État-providence. Les personnes âgées doivent vivre le plus longtemps possible, sans nécessairement bénéficier de ressources financières et matérielles, et surtout sans avoir de formation susceptible de favoriser leur autonomie à mesure qu'elles vieillissent. Ainsi, il devient pratiquement impossible d'atteindre cet objectif.Afin de surmonter ce problème, les personnes âgées se doivent d'apprendre à travailler avec d'autres personnes et de former des partenariats qui leur permettront de garder ou de maintenir leur niveau d'autonomie. 


\section{Recherche sur Internet, niveau 1}

par

\section{Denise Lemire}

Cet atelier s'adresse aux femmes qui ne sont pas familières avec Internet et toutes ses composantes. Les objectifs de l'atelier sont de familiariser les femmes au vocabulaire de Internet, à ses diverses composantes (serveur, navigateur, engin de recherche, etc.) et de permettre quelques expériences de recherche sur l'autoroute de l'information. L'atelier permettra aussi de remettre des renseignements sur les divers sites de la francophonie et plus particulièrement sur les sites liés aux femmes.

\section{Partenariat: un défi à la construction de rapports égalitaires}

par

\section{Lucie Bélanger}

Qu'il s'agisse d'aborder le partenariat par le champ de la recherche ou par celui des pratiques, des enjeux de fond sont à chaque fois en cause, selon des intensités diverses, et ce quel que soit le cadre de mise en oeuvre de son action: le «choc des cultures», soit les arrimages entre les diverses cultures organisationnelles autour de la table, la tension entre efficacité et démocratie, le rapport au temps; le "choc des savoirs», soit la reconnaissance du savoir porté par chaque groupe partenaire et le questionnement de la mission «traditionnelle» de scientificité, l'approche "hiérarchique» des savoirs en regard de la mise en position d'apprentissage pour chacune et chacun des partenaires; le "nerf de la guerre», soit l'attribution des fonds et la structure décisionnelle qui doivent être négociés puis acceptés par l'ensemble des groupes. Les 
partenaires ne sauraient préjuger d'un a priori égalitaire. Il s'agit d'un défi qui exige une vigilance constante, en même temps que la construction de rapports égalitaires est la maitrise de la logique partenariale.

\section{Femmes vieillissantes victimes de violence: une réalité à part?}

par

\section{Céline Simard}

Les femmes victimes de violence vivent dans la peur. Pourtant, celles qui ont choisi de quitter leur conjoint sont nombreuses à retourner dans la relation. Les raisons qui portent les femmes à retourner ou à demeurer dans la relation de violence intéressent les chercheures depuis plus de vingt ans. Pour les femmes vieillissantes victimes de violence, les raisons pour demeurer dans la relation sont-elles différentes? S'expliquent-elles d'une autre façon? Leurs besoins en terme de services sont-ils différents de leurs consoeurs plus jeunes? C'est à partir de ces questions que se déroulera la conférence.

\section{Intervention féministe en maison d'hébergement}

par

Anne Hodge

Les hébergements pour les femmes abusées et leurs enfants sont reconnus pour mettre de l'avant la pratique de l'intervention féministe. Il s'agit d'une intervention qui préconise la prise de conscience, la déculpabilisation des femmes, le choix, la dévictimisation et le changement individuel aussi bien que social. 
Cette intervention vise à aider les femmes à créer des liens entre elles, à travailler ensemble de façon égalitaire, afin de résister à l'oppression systémique du patriarcat. Il est également reconnu que la violence faite aux femmes n'est qu'une des formes d'oppression systémique qui affectent les femmes. Le but de l'intervention féministe est de permettre aux femmes de développer une conscience collective de leurs oppressions et de souligner leur caractère politique. Au fil des années, l'intervention féministe en hébergement s'est élargie afin de mieux répondre à la grande variété des besoins des femmes. Cette intervention confronte de plus en plus les structures et les impacts du racisme, de l'homophobie et des barrières d'âge ou d'habiletés physiques, etc., et reconnait la richesse des différences. Des partenariats ont été créés afin de mieux répondre aux besoins variés des femmes. En prenant la Maison d'amitié comme exemple,j'aimerais explorer l'évolution de l'intervention, les dilemmes et les contradictions qui se présentent, l'importance des partenariats qu'elle a créés, ainsi que l'impact du gouvernement Harris sur l'hébergement et l'intervention.

\section{Femmes en Église : visibles et partenaires?}

par

Diane Dupont

Ces dernières années, beaucoup d'encre a coulé sur la place des femmes en Église. À l'unanimité, on arrive à cette conclusion: malgré les beaux discours sur l'égalité fondamentale des hommes et des femmes, les structures ecclésiales continuent d'exclure les femmes de certaines fonctions relatives aux ministères ordonnés. En 1995, une recherche-action menée dans les diocèses du Québec et de l'Ontario français a abordé la question dans une autre perspective, à savoir les pratiques des femmes en Église et les enjeux qui en découlent. Dans le cadre de ce colloque, nous nous inspirerons de la publication de cette recherche-action, Voix de 
femmes, voies de passage, pour repérer comment les femmes en Église sont visibles et partenaires.

\title{
Intégration, parrainage et réfugiées africaines
}

\author{
par
}

Marie-Béatrice Ngaba et Anne-Marie Mboma

Exposé sur les péripéties du parrainage, source de violence et d'esclavage des personnes parrainées, ainsi que sur les stratégies pour s'en sortir et les stratégies utilisées dans la recherche des voies et des moyens conduisant à l'élimination de la dépendance et à l'acquisition de l'autonomie financière des femmes noires francophones. On verra les stratégies envisagées pour relever le grand défi que représente l'élimination de la dépendance et on soulignera l'importance que revêt l'interdépendance et la solidarité entre tous les groupes minoritaires pour la réussite de cette mission. Finalement, on touchera à l'accueil et à l'intégration des réfugiées dans la région de Hamilton, ainsi qu'aux stratégies utilisées pour relever les multiples défis dans l'accomplissement de leurs responsabilités.

\section{Transformations récentes de la paternité et de la maternité : analyse en référence au cas de la garde physique partagée}

par

\section{Denyse Côté}

La maternité et la paternité se sont construites jusqu'à présent de façon parallèle, complémentaire et asymétrique sur la base de la 
nécessité pour des parents de prendre soin des enfants pendant une période prolongée. Il est donc essentiel de les aborder de façon parallèle. Quelles sont les transformations actuelles de la maternité et de la paternité? Quelles sont les configurations actuelles et quelles seront les configurations futures de ce nouveau tandem? Dans quelle mesure les transformations actuelles sontelles garantes d'un nouveau partage symétrique des responsabilités parentales et du travail de soin des enfants? Cette communication suggérera certaines pistes d'analyse à partir d'une recherche sur la symétrie des rôles parentaux en garde physique partagée.

\section{Logement : outil de prévention ou comment tenir le coup sans retourner dans une situation de violence}

par

\section{Lise Ladouceur}

Lorsqu'une femme décide de sortir d'une situation de violence, elle doit prendre plusieurs décisions qui vont influencer sa qualité de vie et celle de ses enfants. Une de ses premières préoccupations est de se trouver un logement sécuritaire et abordable. Mais où vont ces femmes? Quelles sont les difficultés rencontrées? Quelles sont les options qui leur sont proposées? Quels sont leurs véritables choix? En Ontario, la présente conjoncture socio-économique et politique rend la situation des femmes qui désirent briser le cycle de la violence de plus en plus précaire. Alors, comment prévenir que les femmes retournent chez leur conjoint, ou qu'elles se retrouvent dans une nouvelle relation intime violente à cause d'un manque de ressources financières, de logement adéquat et de ressources communautaires? 


\section{Expérimentation du modèle de thérapie brève axée sur les solutions dans le traitement de cas de survivantes d'inceste}

par

Michelle Bernier-Wilson

La conférencière présentera l'expérimentation du modèle d'Yvonne Dolan, modèle de la thérapie brève axée sur les solutions, dans le traitement de survivantes d'inceste. La pratique des modèles traditionnels, utilisés présentement dans le domaine, est remise en question à travers une réflexion personnelle de mon évolution comme intervenante. Le modèle de la thérapie brève axée sur les solutions et les modèles théoriques ayant contribué à cette approche, celui du stress post-traumatique et celui de l'hypnose naturaliste de Wilton Grickson, sera présenté et appliqué à la symptomatologie de l'abus sexuel. Partant d'une revue de la littérature clinique sur la contribution de différents facteurs relatifs aux résultats de la thérapie, l'approche de Dolan sera comparée aux éléments génériques de la psychothérapie prouvés efficaces. 